\title{
Visualizing the Process of Forming a Shock Pulse in the Deformation Zone
}

\author{
A.V. Kirichek ${ }^{1}$, S.V. Barinov ${ }^{2}$, M.N. Ryzhkova ${ }^{2}$, A.V. Yashin ${ }^{2}$ \\ avk@tu-bryansk.ru |box64@rambler.ru \\ ${ }^{1}$ BSTU, Bryansk, Russia; \\ ${ }^{2}$ VLSU, Vladimir, Russia
}

\begin{abstract}
The article raises the problem of visualizing fleeting processes occurring as a result of wave strain hardening (WSH). The features of this method are unique capabilities for controlling the parameters of the shock pulse. This allows, in contrast to other dynamic methods of the surface plastic deformation, forming the desired microhardness distribution diagram in the surface layer at a depth of 6$8 \mathrm{~mm}$, while ensuring the required uniformity of hardening. The need to visualize this method is explained by the complexity of the analytical description of the ongoing wave processes in the shock system and the loading medium. Developing a visualization technique based on a model of the process of wave strain hardening consists of several stages. The stages include setting the initial and boundary conditions of the simulated elements, their physical-mechanical properties, loading conditions, the type of the mesh, the process conditions. The created model allows you to visually track the shock pulse movement after the striker hits the statically pressed waveguide against the loading medium, and at the same time to see the generation of the reflected deformation wave (the tail of the shock pulse) and its effect on the shock system elements and the loading medium. The results will make it possible to develop shock systems with the highest efficiency.
\end{abstract}

Key words: finite element method, wave strain hardening, visualizing of simulation.

\section{Introduction}

To improve the efficiency and reliability of machine parts in mechanical engineering technology, methods of surface plastic deformation (SPD) are successfully applied [6]. It is known that from an energetic point of view, the dynamic force during SPD is more advantageous than the static force, especially when forming a large hardening depth. At the same time, a significant drawback of dynamic (impact) methods is that the impact energy is spent on plastic deformation irrationally. This is due to the fact that, increasing the loading speed, the flow time of plastic deformation decreases, therefore its value will depend not only on the impact force, i.e. the amplitude of the shock pulse, but also on its duration, which has not usually been considered before when hardening using SPD [1-2]. Accounting for this fact became possible with the advent of wave strain hardening (WSH). A feature of the method is generating deformation waves by a shock system with an intermediate link and their communication to the deformation zone to strengthen critical surfaces of machine parts. The method effectiveness is ensured by a more complete use of the energy of the deformation wave in the hardening process $[3,4,7]$. This is achieved due to preliminary static preloading of the tool-waveguide system to the surface to be treated and subsequent dynamic loading by the striker. Static preloading provides recovery of the reflected deformation waves. As a result of processing, the depth of the hardened layer is 6-8 $\mathrm{mm}$, and the degree of hardening reaches $150 \%$ [5].

Since the WSH process has been developed quite recently, most studies were carried out only experimentally. For comprehensive understanding of the WSH process, it is necessary to know in detail what occurs in the shock system after striking the statically pressed waveguide against the loading medium, how the deformation wave is formed, which affects the energy transfer of the shock pulse. Solving these tasks is possible only in combination, this is due to the fact that the factors under consideration are interconnected and affect each other. It is best to visually evaluate the degree of influence of one or another factor on forming a shock pulse.

The aim of this study is to develop a methodology for visualizing the process of wave strain hardening, based on the model of forming a shock pulse in the deformation zone, using the tools of the modern engineering analysis.

\section{Visualizing the formation of a shock pulse in the deformation zone}

For visualizing fleeting shock processes (of the order of $10^{-5}$ s), the finite element method has proven itself [8-10]. The tools of the modern engineering analysis are based on the principle of this method. The leader in this area is the software package Ansys.

Developing a visualization technique, based on the model of a shock pulse in a deformation zone, is divided into four stages in the process of wave strain hardening.

At the first stage, in the Workbench section of the Ansys program, model elements are created in the graphics module: a striker, a waveguide and loading medium. The striker and the waveguide are rods with flat ends, and the loading medium has the form of a rectangle. Then they are positioned relative to each other, according to Figure 1. To significantly speed up the calculation using the Split Body element, the model is cut along the symmetry axis and only half of the model is left for calculation.

Then, in the Engineering Data module, models of the materials of the shock system and the loading medium are created. The basis for this is the value of the physical-mechanical properties of real materials obtained as a result of the preliminary laboratory studies using the appropriate equipment. In the Symmetry Region section, the boundary of the model symmetry plane is indicated. The breakdown of the created model into finite elements - tetrahedrons (triangular pyramids) is performed in the Mesh section. There, the type of mesh is selected and its size is set. In the Contacts section, the type of Frictional contact between model elements is set and the value of the coefficient of friction is indicated. Using the Contact Tool, contact interactions between model elements are generated.

At the second stage, the Static Structural module sets the preliminary static compression of the loading medium by the waveguide. At the beginning, to limit the displacement of the loading medium under load, the boundary condition Fixed Support is applied to its lower end. The boundary condition Displacement introduces a ban on moving the waveguide along the $\mathrm{Z}$ axis, which avoids its displacement, under load, from the symmetry axis. To account for the effect of gravitational forces on model elements, the Standard Earth Gravity module is added to the calculation. To set the value of the static load, in the Force section, the waveguide is assigned the value of the force of its preloading to the loading medium measured in Newtons. The 
final setup of this stage is completed in the Analysis Settings section by setting the duration of the static loading process.

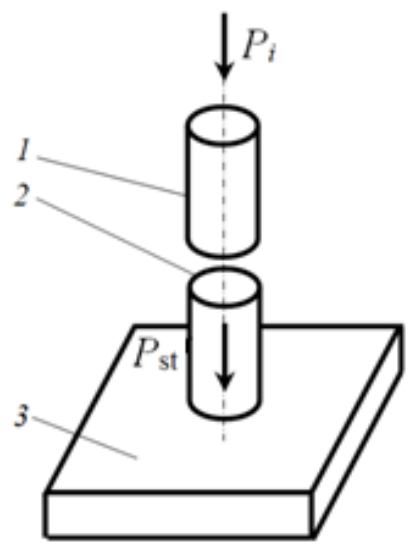

Fig. 1. The scheme of the shock system:

1 - striker; 2- waveguide; 3- loading medium (sample).

At the third stage, the simulation of fleeting shock loading in the LS-Dyna module takes place. As in the previous stage, the model setup begins with assigning the boundary conditions: Fixed Support is a ban on moving the lower end of the loading medium and Displacement is a ban on displacing the striker and waveguide relative to the symmetry axis. The effect of gravitational forces on model elements is taken into account by adding the Standard Earth Gravity module to the calculation. The addition of the Dynamic Relaxation function is necessary to consider in computing the effect of the preliminary static load on the loading medium, calculated in the Static Structural module. The action of the shock load of the striker is realized through the speed in the Velocity section, which is more correct from the energy point of view than the task given through the force parameter. In the Analysis Settings section, the value of the End Time parameter is configured. This parameter includes the sum of the time of static loading, impact and unloading after the impact.

At the fourth stage, in the Solution module, processing and visualizing the simulation results takes place. The module allows you to visualize in the model the actions of various types of deformations, stresses, etc

The developed technique made it possible to visually assess the propagation of the deformation wave in the shock system and the loading medium in the case of a single striking. Visualizing the shock pulse propagation in the elements of the model for a time equal to the period of the shock pulse duration is shown in Figure 2.

\section{Conclusion}

The developed visualization technique, based on the model of shock pulse propagation, will allow you not only to identify the features of its propagation in different types of shock systems, but also to establish their types that will more fully transfer the shock load to the loading medium. The data obtained on propagating the shock pulse in the elements of the shock systems and the loading medium will allow selecting more efficiently the elements of the shock systems for specific hardening tasks.

\section{Acknowledgments}

The reported study was funded by RFBR according to the research project No. 18-38-20066.
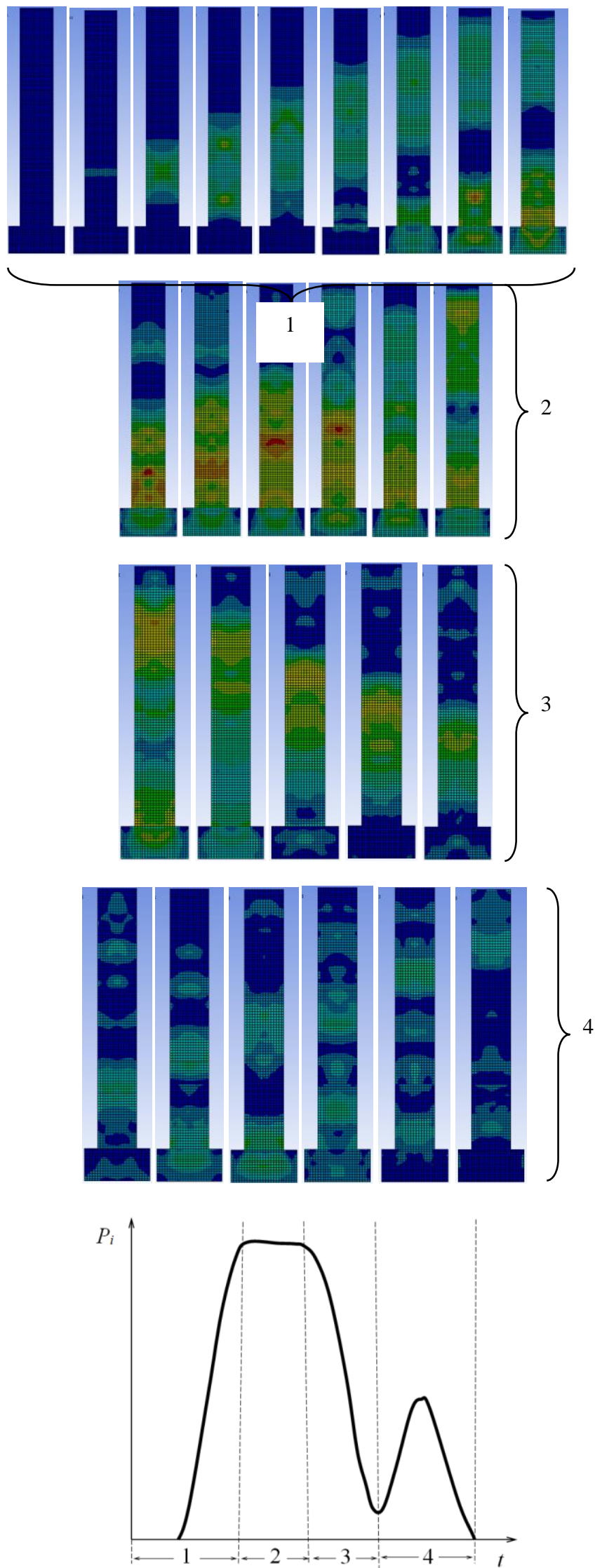

Fig. 2. Shock pulse:

1 - rise front; 2 - period of the shock pulse action; 3 - front of recession; 4 - repeated action of the reflected shock pulse (tail). 


\section{References}

[1] Eremjants V.E., Niu V.V. Two-layer plate stress condition under longitudional impact by tools resting on the plate // Modern problems of theory of mashines. North Charleston. GreateSpace 2016. № 4(1). P. 123-127.

[2] Eremyants V.E., Nju V.V. About selection of parameters of hydraulic vibro-impact machines for surface cleaning // Journal of Advanced Research in Technical Science. 2016. Issue 2. P. 20-24.

[3] Hustrulid W.A., Fairhurst C. A theoretical and experimental study of the percussive drilling of rock // Intern. J. Rock Mech. and Mining Sci., 1971, № 8. - P. 311-333.

[4] Hustrulid W.A., Fairhurst C. A theoretical and experimental study of the percussive drilling of rock // Intern. J. Rock Mech. and Mining Sci., 1972, № 9. - P. 335-356.

[5] Kirichek A.V., Barinov S.V., Aborkin A.V., Yashin A.V., Zaicev A.A. Strengthening of the surface layer of the material by wave deformation multi-contact loading, Materials Science and Engineering, 2017.

[6] Kirichek A.V., Barinov S.V., Yashin A.V., "Patterns of Wave Deformational Multicontact Half-Subspace Loading", Key Engineering Materials, Vol. 736, pp. 12-17, 2017. DOI 10.4028/www.scientific.net/KEM.736.12.

[7] Lundberg B. Some basic problems in percussive rock destruction. - Geteborg, 1971.

[8] Mechanics of plastic deformation in metal processing / Erich Gottfried Thomsen, Charles T. Yang, Shiro Kobayashi. 1965. - $486 \mathrm{p}$.

[9] Shiro Kobayashi, Soo-Ik Oh, Taylan Altan, Metal Forming And The Finite Element Method / New York: Oxford University Press, 1989. ISBN: 0-19-504402-9. $377 \mathrm{p}$

[10] Strang G. and Fix G. J., "An Analysis of the Finite Element Method,” Prentice-Hall, Inc., Englewood Cliffs, 1973. 